\title{
Primary Intraparenchymal Central Nervous System Solitary Fibrous Tumor/Hemangiopericytoma Presenting with Intracerebral Hemorrhage: A Case Report
}

\author{
Myeong-Hun Ha' ${ }^{1}$,Tae-Young Jung ${ }^{1}$, Seul-Kee Kim², Kyung-Hwa Lee ${ }^{3}$, Daru Kim³ \\ Departments of ${ }^{1}$ Neurosurgery, ${ }^{3}$ Pathology, Chonnam National University Medical School, Chonnam National University Hwasun Hospital, \\ Hwasun, Korea \\ 2Department of Pathology, Chonnam National University Medical School, Chonnam National University Hospital, Gwangju, Korea
}

Received October 19, 2018

Revised February 21, 2019

Accepted March 11, 2019

\section{Correspondence}

Tae-Young Jung

Department of Neurosurgery,

Chonnam National University

Hwasun Hospital, 322 Seoyang-ro,

Hwasun-eup, Hwasun 58128, Korea

Tel: +82-61-379-7666

Fax: +82-61-379-7673

E-mail: jung-ty@chonnam.ac.kr
A 53-year old man who had a left hemiparesis from head injury of traffic accident 20 years ago visited an emergency room with suddenly developed semi-comatose mental status. Brain CT showed 8.6-cm sized solid and cystic mass on right temporal lobe that was associated with hemorrhage. Solid lesion showed a strong enhancement after an administration of contrast media. Because of severe mass effect, emergency operation was performed. The mass was an intraparenchymal lesion with yellowish cystic fluid and the firm reddish-brown solid lesion was hemorrhagic. The lesion was totally resected. Pathologically, anaplastic solitary fibrous tumor/hemangiopericytoma was diagnosed with 70/10 high power fields. Postoperative radiotherapy of 50 Gy was done. Postoperative 2 months later, the patient was recovered to alert mental state. We report this unusual case of non-dural based intraparenchymal solitary fibrous tumor/hemangiopericytoma with high mitotic index and acute massive hemorrhage. Rapid tumor growth of hypervascular tumor might have a chance of bleeding.

Key Words Solitary fibrous tumors; Hemangiopericytoma; Hemorrhage; Brain neoplasms

\section{INTRODUCTION}

Hemangiopericytoma is thought to originate from Zimmerman perivascular cells around the capillary [1]. This tumor is rare, accounting for less than $1 \%$ of all primary intracranial tumors. It most commonly affects adults in the fourth to sixth decade of life in males [2]. Most solitary fibrous tumor/hemangiopericytomas are often dural-based. Skull base, parasagittal, and falcine locations are especially common. Only a very small number of cases have reported that tumors occur in the intraparenchymal area [3]. Intracranial hemorrhage is a rare complication in hemangiopericytomas. Such hemorrhage has been explained by hypervascularity and fragile vessels in tumor.

Although soft tissue pathologists have avoided the designa-

This is an Open Access article distributed under the terms of the Creative Commons Attribution Non-Commercial License (https://creativecommons.org/licenses/by-nc/4.0) which permits unrestricted non-commercial use, distribution, and reproduction in any medium, provided the original work is properly cited.

Copyright (๑) 2019 The Korean Brain Tumor Society, The Korean Society for NeuroOncology, and The Korean Society for Pediatric Neuro-Oncology tion of hemangiopericytoma to diagnose these tumors in the spectrum of solitary fibrous tumors, neuropathologists have shown a clear clinical pathologic correlation between hemangiopericytomas and poor clinical behaviors. Solitary fibrous tumor phenotype is generally benign. Gross total resection can be achieved. However, tumors with hemangiopericytoma phenotype have a high rate of recurrence. They may develop extracranial metastases, especially bones, lungs, and liver. Solitary fibrous tumors and hemangiopericytomas have been found to be identical in genetic change. For this reason, the 2016 World Health Organization (WHO) classification of tumors of the central nervous system (CNS) created the term solitary fibrous tumors/hemangiopericytoma to explain these lesions [4].

We report an unusual case of non-dural based intraparenchymal solitary fibrous tumor/hemangiopericytoma with high mitotic index and acute massive hemorrhage. 


\section{CASE REPORT}

A 53-year old man visited the emergency room with suddenly developed mental deepening. On past history, he had an uncontrolled hypertension with left hemiparesis due to head injury of traffic accident 20 years ago. On neurological examination, he showed semi-comatose mental status. Glasgow Coma Scale was 5 . He could not open his eyes. He was withdrawal to pain. He was in intubated state. The pupil size was $8 \mathrm{~mm}$ on right eye and $3 \mathrm{~mm}$ on left. The light reflex was absent on both pupils. The corneal reflex was present. Brain CT showed 8.6-cm-sized solid and cystic mass on right temporal lobe that was associated with hemorrhage (Fig. 1A). Solid lesion showed a strong enhancement after an administration of contrast media (Fig. 1B). The mass effect was severe with 1.8 $\mathrm{cm}$-midline shift from right side to left. The provisional diagnosis was high grade glioma associated with acute hemorrhage. Because of his poor neurological status and severe mass effect, emergency craniotomy and tumor removal were performed. Intraoperatively, the mass was an intraparenchymal lesion without dural attachment (Fig. 1C). The lesion had a cyst with yellowish fluid and myxoid change. It was acute hematoma and firm solid lesion. The solid hemorrhagic lesion contained many vasculatures that were relatively well-circumscribed from normal white mater. The mass was totally resected. Postoperative MRI showed no enhancing lesion on right temporal lobe. Postoperative 3 weeks later, he was recovered to confused mental status with dysarthria.
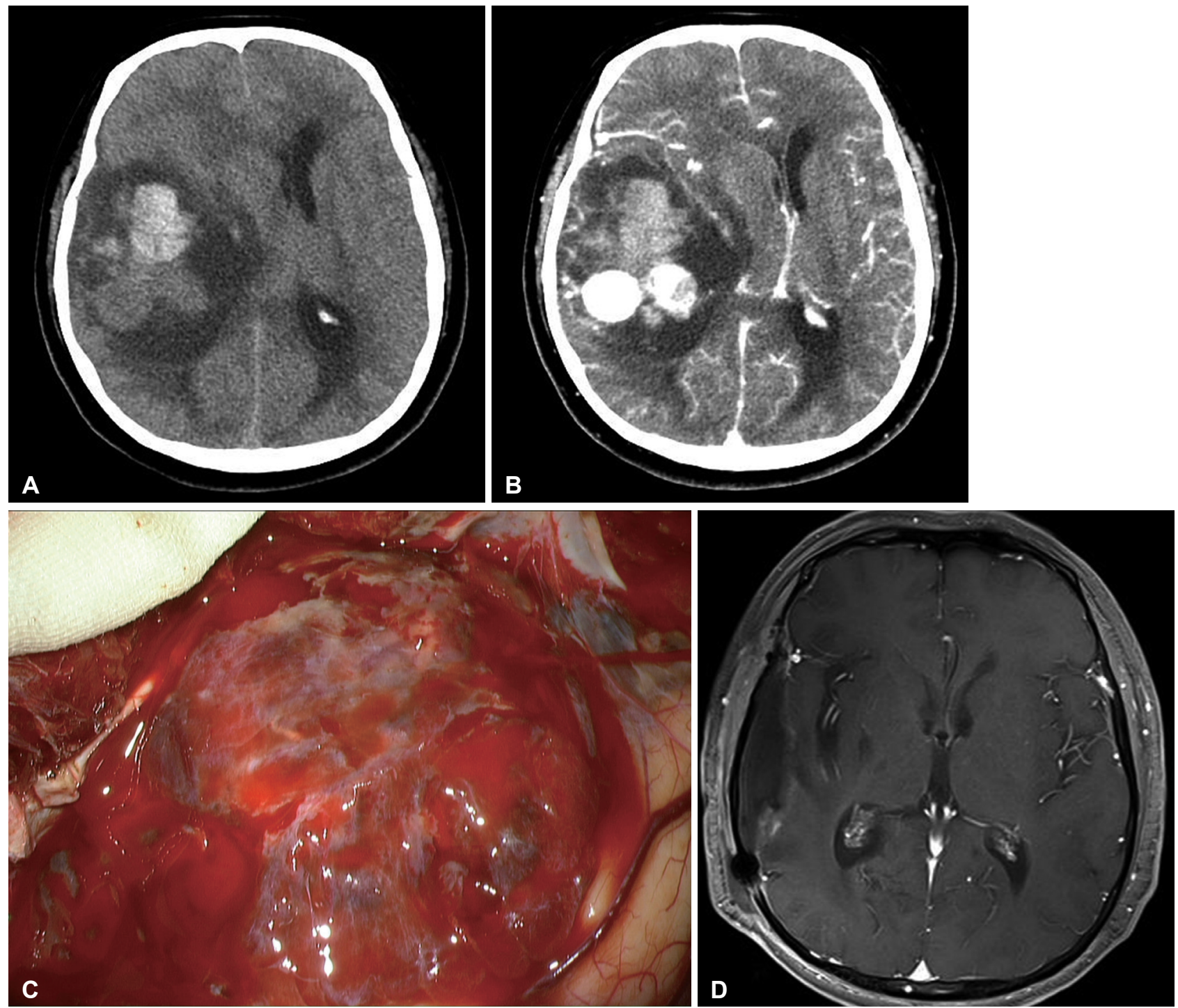

Fig. 1. Radiologic and operative findings. A: Preoperative brain CT shows 8.6-cm-sized solid and cystic mass on right temporal lobe that is associated with hemorrhage and severe mass effect. B: The solid lesion is strongly enhanced after an administration of contrast media. C: Intraoperatively, the mass is an intraparenchymal hemorrhagic lesion with non-dural origin. D: Postoperative 4 months later, MRI shows no enhancing lesion on the right temporal lobe. 

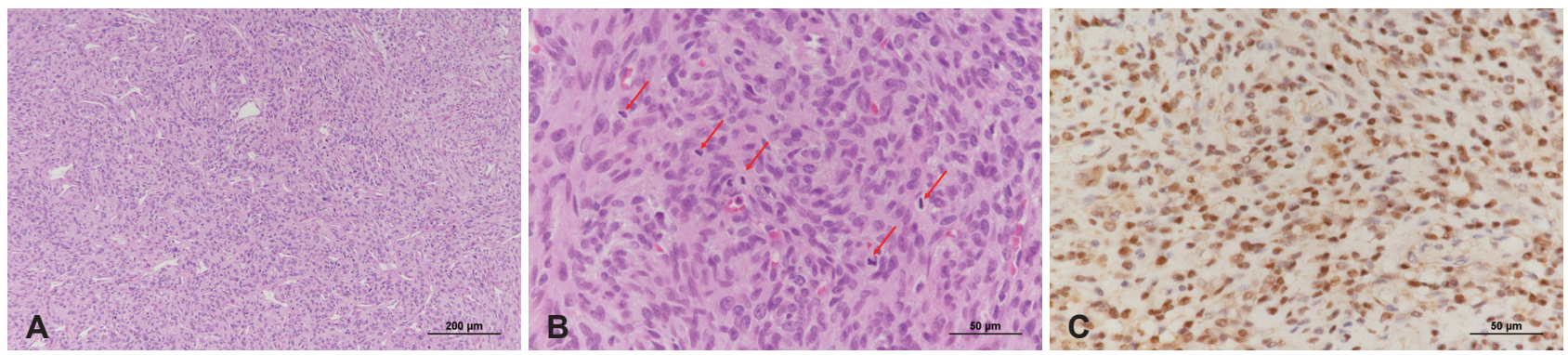

Fig. 2. Pathologic findings of anaplastic solitary fibrous tumor/hemangiopericytoma. Hypocellular and hypercellular areas are divided by hyalinized band with staghorn-like vasculatures (hematoxylin and eosin staining, original magnification $\times 100)(A)$. They show high mitotic index with 70/10 high power fields (red arrows: mitoses, hematoxylin and eosin staining, original magnification $\times 400)(B)$ and immunopositivity for STAT6 (immunohistochemistry, original magnification $\times 400)(C)$.

Pathologically, tumors showed a patternless arrangement. Both the hypocellular area and the hypercellular area appeared. The hyalinized band divided the boundary between hypocellular and hypercellular areas. It was surrounded by thin walled staghorn-like vasculatures (Fig. 2A). Other portions consisted of tissues arranged very tightly which meant solid portion, which had more cellularity and much more dense structures. In random arrangement, stromal surrounding cells were much less while mitosis and necrosis were more common. Mitosis exceeded 70 in 10 high power fields (Fig. 2B). Immunohistochemistry showed STAT6 expression restricted in the nucleus (Fig. 2C). Anaplastic solitary fibrous tumor/hemangiopericytoma with high mitotic index was diagnosed. Postoperative radiotherapy of 5,000 cGy was done. Postoperative 4 months later, the patient was recovered to alert mental state. Brain MRI showed no recurred lesion (Fig. 1D).

\section{DISCUSSION}

Although soft tissue pathologists have diagnosed hemangiopericytoma within the spectrum of solitary fibrous tumors, neuropathologists have suggested retained term of hemangiopericytoma due to distinct clinicopathologic correlations such as high recurrence rate and high systemic metastatic probability. However, both solitary fibrous tumors and hemangiopericytomas share inversion at 12q13 fused with NAB2 and STAT6 genes. Immunohistochemistry can be performed to direct STAT6 nuclear expression. Thus, solitary fibrous tumors and hemangiopericytomas are found genetically identical. For this reason, the 2016 WHO CNS classification established a combined term of solitary fibrous tumor/hemangiopericytoma with a three-tiered system [4]. Grade I tumors consider benign previously diagnosed as solitary fibrous tumor while grade II and III tumors consider malignant previously diagnosed as hemangiopericytoma. Hemangiopericytomas are subclassified as grade II or III depending on mitosis. Solitary fibrous tumor/hemangiopericytoma has two distinct morphological phenotypes. The classic solitary fibrous tumor phe- notype is characterized by the combination of hypocellular and hypercellular areas separated by thick hyalinized bands an. It contains thin-walled branching staghorn vessels. Mitoses area is generally scarce. However, the classic hemangiopericytoma phenotype shows high cellularity and a rich network of reticulin fibers. Mitotic activity and necrosis are often present. Mitotic count is an independent prognostic factor. This case showed high mitotic index.

Hemangiopericytomas are thought to grow on blood vessels and arise pericytes around the capillary [1]. Most hemangiopericytomas are located in the supratentorial area. They are dural based lesion adjacent to falx, tentorium, dural sinus, and skull base [2]. Their radiologic features are similar to meningioma. Different from meningioma, hemangiopericytomas do not show calcifications or hyperostosis of the skull. Unusually, the tumor can be located in lateral ventricle, pineal lesion, and intraparenchymal area [5]. Radiologic findings of this case were subcortically located, heterogeneously enhanced, and relatively well-circumscribed mass with perilesional edema which resembled high grade glioma or metastasis. One postulation of intraparenchymal hemangiopericytoma explained that blood vessels in subarachnoid space adjacent sylvian fissure could be the origin of intraparenchymal hemangiopericytoma. This lesion mimics an intraparenchymal meningioma without dural attachment. Intraparenchymal meningioma is believed to arise from ectopic meningothelial cells within the stroma of the pia-arachnoid that invest perforating blood vessels. This case was also located in the sylvian fissure related to the middle cerebral artery vessels.

Intracranial hemorrhages including intracerebral, subdural, and subarachnoid hemorrhage have been rarely reported [3]. Histopathologic examination showed that $23.4 \%$ of hemangiopericytomas had microscopically intratumoral hemorrhage [5]. Although these bleeding cannot be explained clearly, known hypotheses include erosion, distortion, and distension of fragile and immature blood vessels by tumor growth, vascular distribution and structural changes of tumor commonly known as staghorn-like vasculatures, and vascular occlu- 
sion and necrosis due to endothelial proliferation. As for cause of hemorrhage in this case, high mitotic index meant rapid tumor growth might have caused fragile and immature vessels within hypervascular tumor. Additionally, he had uncontrolled hypertension as an underlying disease.

Radical resection through current surgery is the main treatment for hemangiopericytomas [2]. In addition, high recurrence rate and high metastasis potential necessitate long-term clinical and radiologic follow-up. Radiotherapy is known to improve progression free survival. Postoperative radiotherapy is generally recommended to reduce the risk of local recurrence and delay recurrence. The recurrence rate of hemangiopericytoma is reported to be $50 \%$ to $80 \%$. The average time to recurrence is 39 months (3.2 years). Metastases are reported in $14 \%$ to $30 \%$ of cases. Main metastases sites are bone, lung, liver, rib, and neck. The mean time to metastasis was 79.2 months from the initial diagnosis.

In conclusion, we report an unusual case of intraparenchymal solitary fibrous tumor/hemangiopericytoma with high mitotic index and acute massive hemorrhage. Rapid tumor growth of hypervascular tumor might have a chance of bleeding.

\section{Conflicts of Interest}

The authors have no potential conflicts of interest.

\section{Acknowledgments}

This study was supported by the Basic Science Research Program through the National Research Foundation of Korea (NRF), funded by the Ministry of Science, ICT \& Future Planning (2017R1A1A1A05001020).

\section{REFERENCES}

1. Stout AP, Murray MR. Hemangiopericytoma: a vascular tumor featuring Zimmermann's pericytes. Ann Surg 1942;116:26-33.

2. Guthrie BL, Ebersold MJ, Scheithauer BW, et al. Meningeal hemangiopericytoma: histopathological features, treatment, and long-term follow-up of 44 cases. Neurosurgery 1989;25:514-22.

3. Shetty PM, Moiyadi AV, Sridhar E. Primary CNS hemangiopericytoma presenting as an intraparenchymal mass--case report and review of literature. Clin Neurol Neurosurg 2010;112:261-4.

4. Louis DN, Perry A, Reifenberger G, et al. The 2016 world health organization classification of tumors of the central nervous system: a summary. Acta Neuropathol 2016;131:803-20.

5. Mena H, Ribas JL, Pezeshkpour GH, et al. Hemangiopericytoma of the central nervous system: a review of 94 cases. Hum Pathol 1991;22:84-91. 